\title{
Review: Biomarkers and Role in the Prediction and Detection of Type 2 Diabetes and Its Complications
}

\author{
Ikpotokin Samuel Osahon*
}

College of Medicine, Ambrose Alli University, Ekpoma Edo State, Nigeria

${ }^{*}$ Corresponding author: Ikpotokin Samuel Osahon, College of Medicine, Ambrose Alli University, Ekpoma Edo State, Nigeria; Tel: + 2348133955627; E-mail: ikpotokinosahon@gmail.com

Received date: April 06, 2017; Accepted date: April 25, 2017; Published date: April 28, 2017

Copyright: (C) 2017 Samuel Ol. This is an open-access article distributed under the terms of the Creative Commons Attribution License, which permits unrestricted use, distribution, and reproduction in any medium, provided the original author and source are credited.

Citation: Samuel OI. Review: Biomarkers and Role in the Prediction and Detection of Type 2 Diabetes and Its Complications. Biomark J. 2017, $3: 2$.

\section{Abstract}

Biomarkers are Novel and Revered Tools and Technologies (NORETs) which have helped previous studies anticipate diverse disease conditions and complications as well as provide similarly close prognosis for them. These NORETs in rare but common studies regarding Type 2 Diabetes (T2D) has helped health care professionals and scientists identify health-worthy indications relating to T2D complications and potential risk factors. Biomarkers in its own way debuting in the diagnosis and prognosis of T2D has provided health care professionals with the basic kits of understanding how these diseases manifest from seemingly mild problems which we often times ignore to complicated problems, also indicating possible causes. In this study, the pathophysiology of T2D was subtly exhausted pointing the focus towards the rapidly modifying metabolic manifestations of T2D as well as its risk complications. This study thus reveals the on-going advancement of biomarkers in fully understanding, identifying, diagnosing and treating T2D.

Keywords: Biomarkers; Diabetes; Type 2 diabetes; Hyperglycaemia; Diabetes; Cardiovascular complications

\section{Introduction}

Diabetes in both developed and developing countries of the world has resulted in severe complications like high blood pressure, cardiovascular complications, renal dysfunction, visual malfunctioning, and even blindness. The prevalence of this disease in question has resulted in excessive spending of finances and resources for nearly adequate solutions.

It is common news worldwide that diabetes had expansively affected at least 380-400 million people in the previous years if not more, and has also resulted to about 4-5 million mortality in the year 2014 only. The persistent occurrence of diabetes in its raw form has been predicted to rise rapidly by 2035 [1].
Type 2 Diabetes, (T2D), a type of diabetes, on the other hand, is as a result of insulin resistance (IR) as well as rapid malfunctioning of the pancreatic $\beta$-cell. The continuous and relentless persistence of T2D have multiplied its spread and effects over time now and this is relatively significant to sedentary lifestyle and unhealthy dieting [2]. In order to determine the root causes of this reeling disease, we must closely examine the genetic and environmental factors. Although some relevant genetic studies have subtly identified some factors relating to the high risks for T2D, they haven't yet provided in details a clear understanding of its biological functions. However, the early identification of this disease is paramount in avoiding other risk factors or complications indicating T2D. So, it is importantly relevant that specific biomarkers be identified for the quick and safe analysis and prediction of this prevalent disease, T2D.

When IR decreases rapidly, there is a reciprocal increase in the production of insulin as well as its continuous presence in the body without been duly utilised. This is usually termed hyperinsulinemia and it un-relentlessly leads to the proliferation of T2D. This will surely occur when the pancreatic $\beta$-cell had suddenly become incompetent in regulating the insulin production as well as glucose concentration [3]. Three studies indicated that IR formed the precursor and therapeutic framework or basis in the disease condition of increased glucose concentration and similarly potentiated risk for cardiovascular disease (CVD) and vascular disposition for T2D occurrences [4-6]. Several biomarkers like the branched-chain amino acid and micro Ribonucleic acids (mRNAs) have been exclusively identified in recent studies into-omics, for T2D $[7,8]$. More so, studies like those of Yang et al. [9] and Barazzoni et al. [10] have provided researchers with potential biomarkers (ghrelin and retinol-binding protein) to work with, yet, Echouffo-Tcheugui et al. [11] could rarely see through the loop into T2D risk complications. It would be an outstanding relief when substantial biological tools in the prediction of T2D and risk complications as well as its relatives are identified. This would in no doubt ease better analysis for the effective prediction of T2D as well as in the administration of relevant diagnosis and prognosis and this was probably in Assarsson's et al. collaborative thinking in providing the Proximity 
Extension Assay (PEA). This positive thinking has played a role in improving the comparative studies of protein in their cumbersome nature within very small samples [12].

Delving into the concept of biomarkers, we find that biomarkers have long painted the walls of qualitative and quantitative research over the years now. Several countries have participated in the biomarker revolution in expressly every biological assay. We discovered from these sole benefactors of the advances of biomarkers in the early ages of research development had noted that the sweet taste, which was actually raw and concentrated indicated the presence of excessive sugar which in turn contributed to the basis for diabetes test using urine. $\mathrm{HbA} 1 \mathrm{c}$, a glycohemoglobin value culminated an important criterion for the distinct regulation of blood sugar levels in both type diabetes (T1D and T2D). This $\mathrm{HbA1c}$ value spontaneously retains the sugar data of the patient in question and processes the acquired data based on the concentration levels for some time, even lasting weeks, but when the blood sugar eventually eases down or better yet improved upon, the elevated values comes falling down.

Ron Zimmer [13], described biomarker as proteins, cell, molecules, genes even enzymes or hormones which could be measured in their own capacity, religiously assessed as a primary indicator for the homeostatic processes and responses aimed at delivering means to solving physiologic complications. Houlka [14] had noted that biomarkers though expressly vague have been a major experimental tool by researchers and scientists to analytically study human diseases. Desai [15] expressly reported that 'blood pressure' though a state, served as a surrogate endpoint biomarker in correlating the adverse effects of elevated/increase blood pressures on cardiovascular output. We definitely can't place all biomarkers as a surrogate end-point identifier, thus making their identification a 'Novel and Revered Tool and Technology (NORET)' of researches into the lavish use of biomarkers in research as well as other analytical studies. In terms of professionalism, biomarker seems to be growing at a slower rate though it had been expressly useful in pre-clinical and clinical studies for a considerable amount of time, and has been a bed for which quality research though adamant, had spread their heart-ached analysis of simpler and complex physiological problem alike.

Body temperature is a comparative and common biomarker for fever. Blood pressure is another comparative and first sought after NORET for stroke indications and complications. Cholesterol values are NORETs indicating coronary and vascular diseases, and C-reactive protein (CRP) is another thought of NORET for inflammation.

Resoundingly, biomarkers may include NORETs that can assess the necessary and vital information concerning a particular problem or complication. Digressing a little, we find that in Perera and Weinstein's and Naylor's studies that as complex and complicated the nervous system is and has been in recent times, the wider and more advanced the NORETS utilized in assessing the information about the Nervous boxthe brain and related disease complications [16,17]. Among such NORETs are assessing the blood or Cerebrospinal Fluid
(CSF). These may involve measurements directly on biological media (e.g., blood or cerebrospinal fluid). The biomarker in its tailored parametric (chemical, physical and biological) capacity can independently assess the growth and development of the disease, likewise the receptive effects of the administered management.

With these whelming explanations made easy, biomarkers are simply any kind of indicator that gives an accurate or near accurate diagnosis of certain physiologic problems and in this study, T2D.

\section{Characteristics of Biomarkers}

\section{Among the labelled characteristics of an ideal biomarker} are:

- Safety and easy to measure (Quality over Quantity-Qua/ Quo).

- Affordability.

- Proven treatment to modify any given set of biomarker(s).

- Consistency irrespective of personality.

- Sensitivity and specificity.

\section{Exceptional predictive value(s)}

Describing briefly the features above, Pharma Matters White Paper, in a comprehensive study noted that in all patients with a disease condition, a positive result can be determined with the specificity and sensitivity of the test [18].

\section{Newman and Unger's criteria for biochemical/molecular biomarkers include:}

- Biomarkers must be measurable at all times [19].

- Biomarkers must have rapid response, inexpensive and easy to use.

- Biomarkers must proffer Qua/Quo.

- Biomarkers should be specific (which in most cases are unlikely impractical).

- Biomarkers should possess a linear concentration-effect relationship.

- Biomarkers should be applicable to similar species.

\section{Biomarkers Requisite}

In determining the biomarker requisite for T2D, I quickly hover around the prevalence of other disease conditions and their identifiable biomarkers for easy navigation through this study. We seldom experience people or individuals with long term illness requiring long term medications with less accurate diagnosis of the persistent conditions especially in African countries and other parts of the world. With the favourable hand of biomarkers and NORETs, diseases can be effectively diagnosed and proffered suitable treatments [20]. Diseases like Alzeimer and rheumatoid arthritis which often times starts off with an undetectable symptom stage until it becomes seriously hurting, in these instances, biomarkers have helped to quickly identify them as well as individuals susceptible to their complications. 
For a biomarker to be successfully utilized, the presented sample material(s) which could be blood sample, urine or even saliva must be fast and like oil drop on a white cloth for easy examination and commencement of treatment. Hence proper consultation process can immediately ensue for patients already diagnosed. A rapid test, which delivers a result after only a few minutes, is optimal. This makes it possible for the physician to discuss with the patient how to proceed and if necessary to start treatment immediately after the test.

\section{Pathophysiology of type 2 diabetes}

Already possessing a glimpse or two about the metabolomics for biomarkers especially for the T2D, I subtly digress into the pathophysiology of this study disease. As important as it is hitting the nail right on point, it is essential I reel in the intrigue behind T2D. We definitely realize that the prevalence of T2D is as a result of progressive depletion in glucose tolerance until it finally results in diabetes. What we both should also remember is that T2D is and can be detected on the basis of sporadically increased HbA1c level, leading to IR and pancreatic $\beta$-cell malfunctioning.

Expressly evaluating older studies, I had realized the basic conspiracy in the pathophysiologic defects in patients with T2D; Impairment, Deterioration, Genetic Re-wiring and Environmental familiarities.

A long time ago, noted that sporadically increased levels of saturated fatty acids (FAs) could damage and continuous the steady secretion of insulin leading to a significantly low glucose tolerance [21]. Supporting Swinburn et al. and Lillioja et al. [22], noted in another comprehensive study, that damaged and continuous secretion of insulin examines T2D; we fill in the blank spaces and proffer effective measures against such defects. In the 70's had noted then that certain impairment in the secretion of insulin are as a result of reduced/deteriorating sensitivity of the glucose $(G)$ receptors responsible for conveying the glucose signal to trigger insulin release [23]. Genetic and environmental factors are the main determinants of insulin secretion. Pimenta et al. [24] confidently noted that the genetic and environmental factors determine the secretion of insulin.

The issues surrounding biomarkers in health and diseases, have expressly increased, yet biomarkers in field applications had face a resilient wall trying to first combat and settle the dispute between statistically effective studies carefully moderating clinical problems and complications $[25,26]$.

\section{Biomarkers for $\mathrm{T} 2 \mathrm{~d}$ prediction}

Generally, predicting diabetes has been of concern to many researchers and scientists all over the world. However there has been some new interventions regarding blood samples as biomarkers becoming even more and more complex by the day, but researchers an scientists need slated laws which points the scientists towards clinically analysing diabetes. In this paper, I delve into some standard areas that had suffered little or no feathered heart concentration as far as T2D and its health complications are concerned. However these areas should not be given a one glance consideration but a nicely dished salad attention. They are as follows:

- First and foremost, we label the severity of T2D to better provide the necessary dispensation for patients/individuals at death's door (stroke/heart attack) by desperately assessing CVD complications, which on its own must be as refined as liquid gold. On the contrary, diagnosis of diabetes which is defined based on a blood sampling analysis for the various complications of diabetes can always be repeated as long as the individual still portrays some persistently increase risk complications. Prediction of T2D in these contrasting situations becomes unfailingly less important or critical.

- In a study, extensively noted and reported that at receiver operating characteristics (ROC) of about 0.80 are possible indicators for diabetes at simplified risk score such as Body mass index (BMI), age, family histoire of diabetes, even age [27]. Noting this, health care professionals can be steered thus to naturally selecting suitable candidates for blood sampling tests for T2D. Still on this note, Wannamethee et al. [28] noted that simple risk scores for diabetes anywhere around the world can be easily determined, and once taken up; the exclamation in the Einstein's light bulb is to determine what the cut-points are for proceeding to a blood test.

- In a 2011 study, Wannamethee et al. [29], also noted that $\mathrm{HbA1c}$ in close combination with other simple parameters under the risk score ROC would better increase and enhance future prediction of diabetes as well as T2D. However, individuals with reduced $\mathrm{HbA} 1 \mathrm{c}$ values should be considered at potential risk of future diabetes as well as T2D, and quickly proffered a composite and conducive lifestyle intervention [2].

- Finally, there is the need to statistically and significantly relate cardiovascular diseases and diabetes risk screening because nowadays, both go hand in hand building bridges (more or less a catch 22 situation). Risk screening and complications of diabetes has been a family chat for health care professionals over every centre table discussion, but rarely has these resolutions by the years been implemented given its ball and chain complexity and the necessity for continuous tests. Where there's a seed, there's hope, and the initiation of $\mathrm{HbA} 1 \mathrm{c}$ as a NORET for the diagnosis and prognosis of diabetes has paved way to further growing this seed of faith to advancing diabetes analysis and checkmating risk complications and identifications. HbAlc testing does not require fasting or denial of food, thus providing room for constant and steady $24 / 7$ screening for diabetes. Afterall, Di Angelantonio [8] had noted that non-fasting lipid reports were presented as would fasting lipid reports for CVD risk prevention. Interestingly, Griffin et al. [12] noted in their study that an earlier screening of diabetes would lesson CVD risk complications and increase the chances of applying better improved lifestyle practices. This suggestion is speculative, but, clearly, picking up diabetes sooner after HbA1c crosses the diagnostic threshold could have multiple advantages for patients. 


\section{Biomarkers for T2d, complications, treatment guidance}

A snail and shell relationship exists between the available diabetes treatment, $\mathrm{HbA} 1 \mathrm{c}$ value indicators, ranging lipid levels, correlating cardiac outcomes, and likely survival. This symbiotic relationship surpasses the available ideas and ideals, placing a limiter to comprehending their interactions and possible workings $[30,31]$.

Scientific assumptions regarding the reliability of biomarker reports on a peculiar disease are sometimes regarded as a half arms' worth. Khaw et al. [32] had assumed in a comprehensive study using the $\mathrm{HbA1c}$ value, that serious regulation of blood glucose would in its own way reduce the risk complications of diabetes as well as T2D complications, as documented by the U.K Prospective Diabetes Study [33]. Due to the swell rates of CVD in patients with T2D, both background and underground studies have tried to correlate the effects of sufficient glycemic control over CVD risks and complications but to no avail [34-36].

\section{Conclusion}

Closely observing the tethering nature of biomarkers and their susceptible efficiency, there is still a lot of work to be accomplished in the field of biomarkers as well as its diagnostic use in the prognosis of diabetes mellitus as well as its risk complications. This study as well as other related studies had shown some of the clinical correlation of biomarkers in the possible identification of T2D. In this study also, we had diversified our minds to the various complications of T2D without highlighting the relative effects in women population. This study shows how possible it is to quickly and safely acquire relevant data on the variant functioning of individual metabolites that rustles glucose tolerance and insulin sensitivity. Here we realize that the relationship between increased glucose concentration and T2D were seriously at loggerheads. The biological features of these related variants have been like searching for a tiny pin in hay stack-challenging. In conclusion, I have identified some NORET biomarkers (e.g. the sweet taste of urine, the receiver operating characteristics (ROC) of about 0.80 and $\mathrm{HbA1C}$ ) that have been used in previous and continuous studies for assessing the risks and development of diabetes, T2D as well as their complications since these NORETs have in times past and continuously help predict the onset of T2D and consequently diagnosis and fast-racing prognosis before the coming rains.

\section{References}

1. International Diabetes Federation (2014) IDF Diabetes Atlas (6th edn.), Brussels Belgium 123: 32-39.

2. Ikpotokin SO, Adeleye SO, Aliyu D, Osayande B, Ehiabhi (2017) Dietary Factors in Fasting Blood Glucose Levels and Weight Gain in Female Sprague Dawley in Rats. J Clin Nutr Diet 3:1.
3. Dali-Youcef N, Mecili M, Ricci R, Andrès E (2013) Metabolic inflammation: connecting obesity and insulin resistance. Ann Med 45:242-253.

4. Bonora E, Formentini G, Calcaterra F, Lombardi S, Marini F (2002) HOMA-estimated insulin resistance is an independent predictor of cardiovascular disease in type 2 diabetic subjects: prospective data from the Verona Diabetes Complications Study. Diabetes Care 25: 1135-1141.

5. Nowak C, Sundström J, Gustafsson S, Giedraitis V, Lind L, et al. (2016) Protein Biomarkers for Insulin Resistance and Type 2 Diabetes Risk in Two Large Community Cohorts. Diabetes 65: 276-284.

6. Park SW, Kim SK, Cho YW, Kim DJ, Song YD, et al. (2009) Insulin resistance and carotid atherosclerosis in patients with type 2 diabetes. Atherosclerosis; 205: 309-313.

7. Batch BC, Hyland K, Svetkey LP (2014) Branch chain amino acids: biomarkers of health and disease. Curr Opin Clin Nutr Metab Care 17: 86-89.

8. Guay C, and Regazzi R (2013) Circulating microRNAs as novel biomarkers for diabetes mellitus. Nat Rev Endocrinol 9: 513-521.

9. Yang Q, Graham TE, Mody N, Preitner F, Peroni OD, et al. (2005) Serum retinol binding protein 4 contributes to insulin resistance in obesity and type 2 diabetes. Nature 436: 356-362.

10. Barazzoni R, Zanetti M, Ferreira C, Vinci P, Pirulli A, et al. (2007). Relationships between desacylated and acylated ghrelin and insulin sensitivity in the metabolic syndrome. J Clin Endocrinol Metab 92: 3935-3940.

11. Echouffo-Tcheugui JB, Dieffenbach SD, Kengne AP (2013) Added value of novel circulating and genetic biomarkers in type 2 diabetes prediction: a systematic review. Diabetes Res Clin Pract 101: 255-269.

12. Assarsson E, Lundberg M, Holmquist G, Björkesten J, Thorsen SB, et al. (2014) Homogenous 96-plex PEA immunoassay exhibiting high sensitivity, specificity, and excellent scalability. PLoS ONE 9: e95192.

13. Ron Zimmer MA (2008) Evaluation and Regulation of Biomarkers A Public Health Perspective PHG Foundation OECD Workshop on Policy Issues for the Development and Use of Biomarkers in Health Wellcome Trust Conference Centre Hinxton..

14. Houlka BS (1990) Overview of biological markers. In: Biological markers in epidemiology Oxford University Press, NY, USA pp: 3-15.

15. Desai M (2006) Blood Pressure as an Example of a Biomarker That Functions as a Surrogate. AAPS J 8: 146-152.

16. Perera FP, Weinstein IB (2000) Molecular epidemiology: recent advances and future directions. Carcinogenesis 21: 517-524.

17. Naylor S (2003) Biomarkers: current perspectives and future prospects. Expert Rev Mol Diagn 3: 525-529.

18. https://www.thomsonreuters.com/content/dam/openweb/ documents/pdf/pharma-life-sciences/white-paper/establishingthe-standards-in-biomarker-research.pdf

19. Newman MC, Unger MA (2002) Fundamentals of ecotoxicology. USA: Lewis publishers.

20. Waaler $E$ (2007) On the occurrence of a factor in human serum activating the specific agglutination of sheep blood corpuscles. APMIS 115: 422-438. 
21. Swinburn BA, Boyce VL, Bergman RN, Howard BV, Bogardus C (1991) Deterioration in carbohydrate metabolism and lipoprotein changes induced by modern, high fat diet in Pima Indians and Caucasians. J Clin Endocrinol Metab 73: 156-165.

22. Lillioja S, Mott DM, Spraul M, Ferraro R, Foley JE, (1993) Insulin resistance and insulin secretory dysfunction as precursors of non-insulin-dependent diabetes mellitus. Prospective studies of Pima Indians. N Engl J Med 329: 1988-1992.

23. Cerasi E, Luft R, Efendic S (1972) Decreased sensitivity of the pancreatic beta cells to glucose in prediabetic and diabetic subjects. A glucose dose-response study. Diabetes 21: 224-234.

24. Pimenta $\mathrm{W}$, Korytkowski $\mathrm{M}$, Mitrakou A, Jenssen T, Yki-Jarvinen $H$ (1995) Pancreatic beta-cell dysfunction as the primary genetic lesion in NIDDM. Evidence from studies in normal glucosetolerant individuals with a first-degree NIDDM relative. JAMA 273: 1855-1861.

25. Ioannidis JP, Panagiotou OA (2011) Comparison of effect sizes associated with biomarkers reported in highly cited individual articles and in subsequent meta-analyses. J Am Med Assoc 305: 2200-2210.

26. Sturgeon C, Selby P (2010) Development and validation of new biomarkers: major opportunities for health-care scientists. Ann Clin Biochem 47: 499-502.

27. Sattar N, Wannamethee SG, Forouhi NG (2008) Novel biochemical risk factors for type 2 diabetes: pathogenic insights or prediction possibilities? Diabetologia 51: 926-940.

28. Wannamethee SG, Papacosta O, Whincup PH, Thomas MC, Carson C, et al. (2011a) The potential for a two-stage diabetes risk algorithm combining non-laboratory-based scores with subsequent routine non-fasting blood tests: results from prospective studies in older men and women. Diabet Med 28: 23-30.
29. Wannamethee SG, Welsh P, Lowe GD, Gudnason V, Di Angelantonio $\mathrm{E}$, et al. (2011b) N-terminal pro-brain natriuretic peptide is a more useful predictor of cardiovascular disease risk than C-reactive protein in older men with and without preexisting cardiovascular disease. J Am Coll Cardiol 58: 56-64.

30. Di Angelantonio E, Sarwar N, Perry P, Kaptoge S, Ray KK, et al. (2009) Major lipids, apolipoproteins, and risk of vascular disease. J Am Med Assoc 302: 1993-2000.

31. Griffin SJ, Borch-Johnsen K, Davies MJ, Khunti K, Rutten GE, et al. (2011) Effect of early intensive multifactorial therapy on 5-year cardiovascular outcomes in individuals with type 2 diabetes detected by screening (ADDITION-Europe): a cluster-randomised trial. Lancet 378: 156-167.

32. Khaw KT, Wareham N, Luben R, Bingham S, Oakes S, et al. (2001) Glycated haemoglobin, diabetes, and mortality in men in Norfolk cohort of European Prospective Investigation of Cancer and Nutrition (EPIC-Norfolk). BMJ 322: 15-18.

33. UK Prospective Diabetes Study (UKPDS) Group (1998) Effect of intensive blood-glucose control with metformin on complications in overweight patients with type 2 diabetes (UKPDS 34). Lancet 352: 854-865.

34. Duckworth W, Abraira C, Moritz T, Reda D, Emanuele N, et al. (2009) Glucose control and vascular complications in veterans with type 2 diabetes. N Engl J Med 360: 129-139.

35. Gerstein HC, Miller ME, Byington RP, Goff DC Jr, Bigger JT, et al. (2008) Action to Control Cardiovascular Risk in Diabetes Study Group, Effects of intensive glucose lowering in type 2 diabetes. N Engl J Med 358: 2545-2559.

36. Patel A, MacMahon S, Chalmers J, Neal B, Billot L, et al. (2008) Intensive blood glucose control and vascular outcomes in patients with type 2 diabetes. N Engl J Med 358: 2560-2572. 